THE MAGNETIC PROPERTIES OF THE SLC INTERSECTION REGION SUPERCONDUCTING QUADRUPOLE TRIPLETS

\author{
A. D. MeInturff, J.A. Carson, H. E. Fisk
}

Fermi Nat Ional Accelerator Laboratory*

P. O. Box 500

Batavia, Illinols 60510

R. A. Erickson

Stanford Linear Accelerator Centert

P. 0 . Box 4349 Bin 21

Stanford, California 94305

Abstract: The measured magnetic field parameters of the quadrupoles which comprise the final triplet. lens system for the the SLAC LInear Collider intersection region are presented here. The minimum design gradlent specifications for these quadrupoles are $1.7 \mathrm{~T} / \mathrm{cm}$ at $4.6 \mathrm{~K}$ and $1.6 \mathrm{~T} / \mathrm{cm}$ at $4.6 \mathrm{~K}$ in a $0.6 \mathrm{~T}$ external solenoldal field. These gradients are about three times larger than those avallable with the conventional 1ron/copper quadrupoles now used in the SLC. Superconducting quadrupnles of two lengths have been speclfled for the SLC triplets. The effective magnet1c length of type $Q$ is $66.498 \pm 0.305 \mathrm{~cm}$ and of $Q_{2}$ is $121.106 \pm 0.61 \mathrm{~cm}$. The superconducting performance characteristics of the quadrupoles that have been measured are: maximum critical current as a function of bath temperature, rate of change of magnetic field, and as a percentage of the "short sample". "Short sample" performance is defined as the current reached by the cable in a perpendicular magnetic fleld equal to the peak field in the winding at bath temperature. The maximum gradient achleved during testing was $2.09 \mathrm{~T} / \mathrm{cm}(4.25 \mathrm{~K})$ and $2.10 \mathrm{~T} / \mathrm{cm}$ (3.2K). This represented $95 \%$ of the strand critical current value. The magnetic length of the rirst $Q_{2}$
was measured to be $120.85 \pm .1 \mathrm{~cm}$. The Fourler harmonic coefficients of the magnetic fleld were measured as a function of current and are reported.

Introduction: A pair of superconducting triplets comprise the proposed superconducting final focus system. The operational gradlent for the elements in the triplets is about $1.35 \mathrm{~T} / \mathrm{cm}$ at a beam energy of 55 GEV. This higher gradlent should increase the luminosity by a factor of 1.6 to 3.2 compared' to the present conventional system. The triplet quadrupoles do not have iron shlelds due to their physical position inside the $0.6 \mathrm{~T}$ solenoldal field of the detector.

\section{Design and Construction}

The design of the SLC triplet quadrupole is. covered in an earlier paper. ${ }^{2}$ The cross-section of the 96 turn winding and collar structure is shown in Figure 1. The ininer winding diameter is five centimeters, and the clear aperture is $4.6 \mathrm{~cm}$. This aperture was dictated by the background radiation requirements of the experimental detector. A smaller aperture would result in unacceptably high rates of secondary scattered electrons. The design of these quadrupoles is basically simflar to the Tevatron quadrupoles, except for the smaller aperture. The magnetic fleld was lowered in the ends of the coll by the addition of six spacers between the tirns nearest.

* Operated by Universitles Research Association, Inc., under contract with the U. S. Department of Energy.

TWork supported by the Department of Energy, contract DE-ACO3-76SF00515.

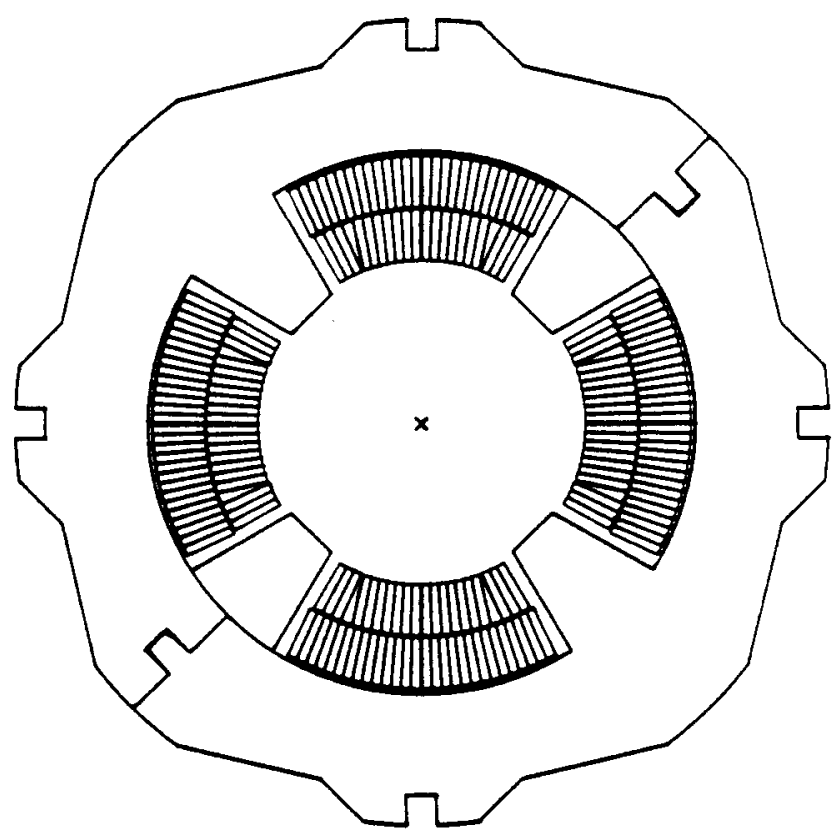

FIGURE 1 THIS IS A DRAWING OF THE CROSS-SECTION OF THE SLC COLLARED COIL ASSEMBLY.

the pole. There was no addittonal attempt to correct the harmonics in the ends of these quadrupoles. This smaller aperture, and the resulting sharp bends in the colls, led to turn-to-turn shorts. This problem was solved in the early stages of construction, as discussed below.

After construction began, a breakdown in Insulation was encountered on the second $120 \mathrm{~cm}$ quadrupole, 4802. The cable Insulation was discovered to have falled at the transition area where the cable makes $1 t^{\prime} s$ way from the 1nner coll to the outer coll. Cable damage was also evident. This same problem caused two additional $120 \mathrm{~cm}$ quadrupoles (\$4800 and 4803 ) which were in the production plpe line to fail. The coll pre-stress for Magnets 4801 and $\$ 4803$ was also noticed to be excessive. All coll winding and assembly was halted at this time. The insulation damage in the transition area was determined to be caused by an unstable cable geometry resulting from the torturous path the cable has to follow in the transition area. This resulted in the cable moving from it's as-wound postition causing interferences and excessive loading during the cofl molding and curing processes. A fixture was developed that soldered the cable int geometrically stable shape in the iranaltion area prior to coll winding. Extra 
insulation was also added at the transition. The result of this "flx" was the virtual ellmination of layer to layer electrical shorts occuring in the windings, both before and after the assembly into a magnet. The small aperture had made the geometry of this transition much more sensitive to shape and rigldity than had previously been seen in Tevatron magnets.

The excessive coll pre-stress encountered in quadrupoles 4802,4803 ; and 4800 was traced to an excessive overlap of the Kapton inner wrap insulation around the cable. This resulted in a build-up during the winding process, which in turn, resulted in high molding and collaring pressures. high molding pressures can lead to insulation creep and result in turn-to-turn shorts. The situation was remedled by Increased quality control during the cable insulating process.

The characteristics of the strand used to fabricate the cable are given in Table I and the cable in Table II.

Table I

Strand Characteristics

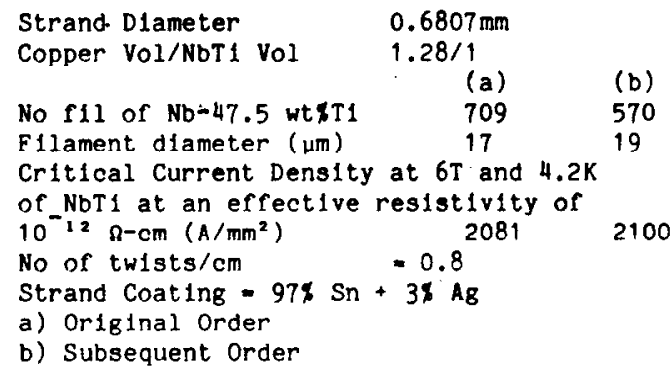

\section{Table II}

'Rutherford' Style Cable* Characterlstics Using Strand from Table 1

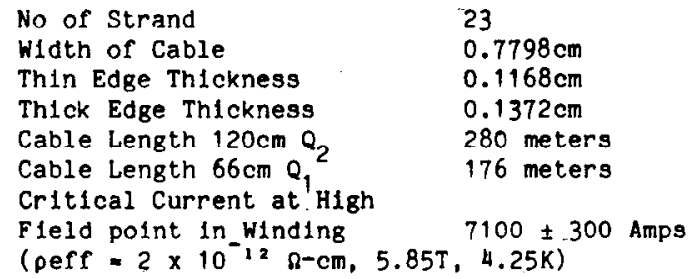

"Note: These are the same physical dimenslons as the "Tevatron" cable.

The individual pole coll stzes in SLQ, 2602, 2603, 2604, 2605, and SLQ 4804 matched to $5.0 .069 \mathrm{~mm}$ which resulted in a reduction of the Fourler coefficients of the magnetic fields produced.

\section{Magnet Performance}

The quadrupoles did show 'training'; 'training' being defined as premature superconducting to normal state resistive transitions occurring during early powering cycles. The varlous training scenarlos could be summarlzed as follows: The first quench usually occurred at or above six klloamperes (Grad. $1.68 \mathrm{~T} / \mathrm{cm})$. For about $\mathrm{f} 1 \mathrm{ve}$ to ten quenches at slow ramp rates ( 2 to 6 amps/sec), the quadrupole would quench at around seven k1loamperes (Grad $=1.96 \mathrm{~T} / \mathrm{cm}$ ) at $4.2 \mathrm{~K}$. Quench histories are shown in Figure 2. See Table A, B, C, and D for actual data.

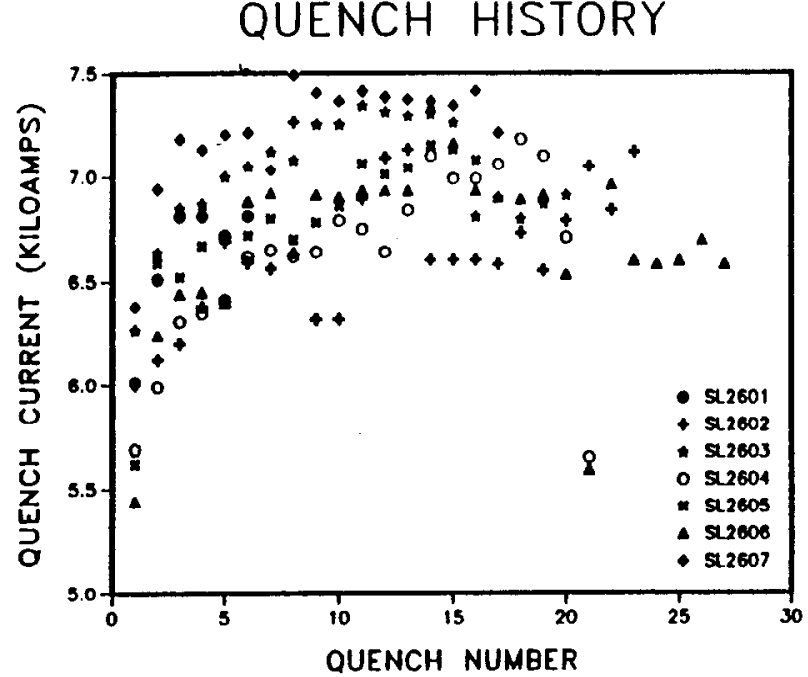

F1gure 2A

\section{QUENCH HISTORY}

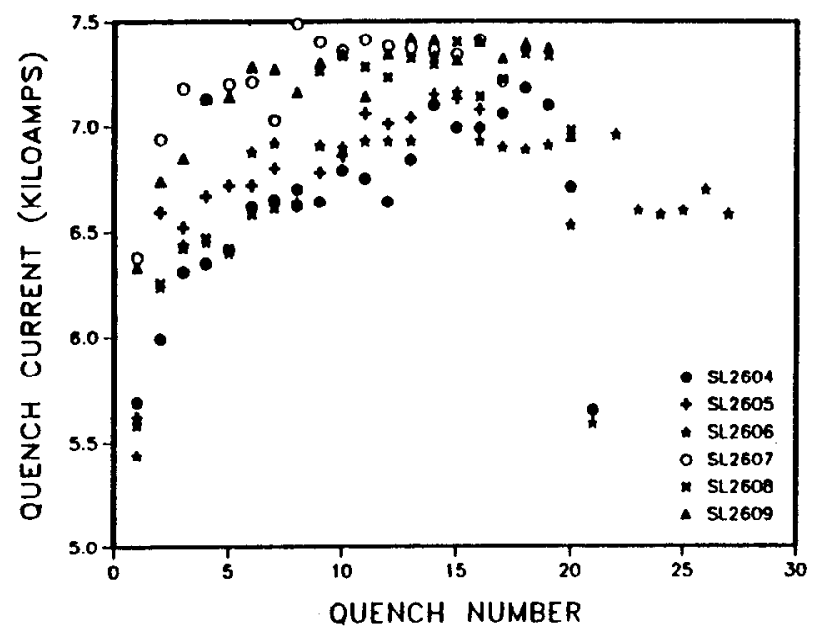

F1gure 2B
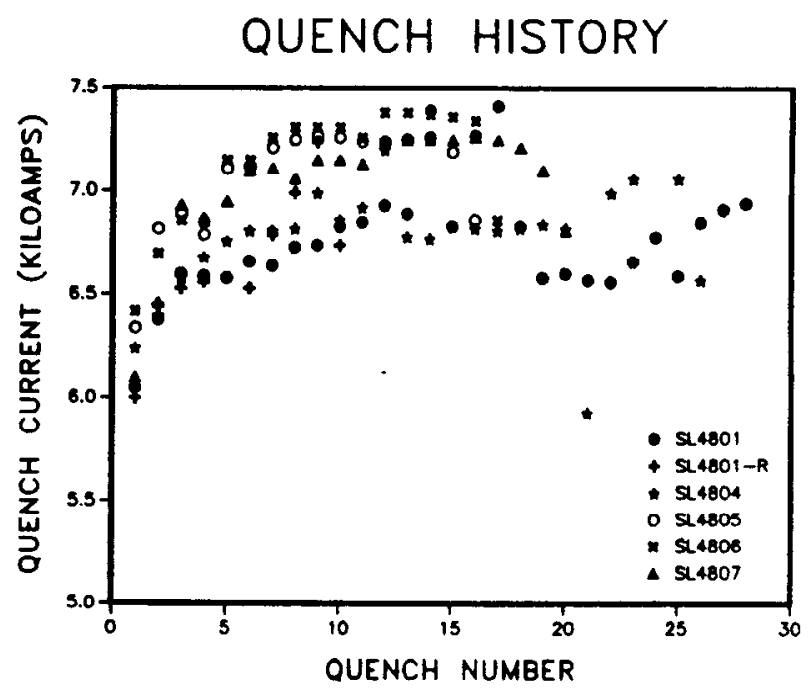

Figure 2C 
The magnets made before the transition fixture was used showed a marked ramp rate dependence. Magnets fabrlcated with the flxture had a much less pronounced ramp rate dependence. This ramp rate dependence can be seen in Figure 3. It is of interest to note that the SLC triplets w111 operate $\mathrm{DC}$ at a gradlent of about $1.4 \mathrm{~T} / \mathrm{cm}$. There is a time dependence to the magnetization contribution of the harmonic content of the magnet field which w1ll be given later.

RAMP RATE DEPENDENCE OF QUENCH CURRENT

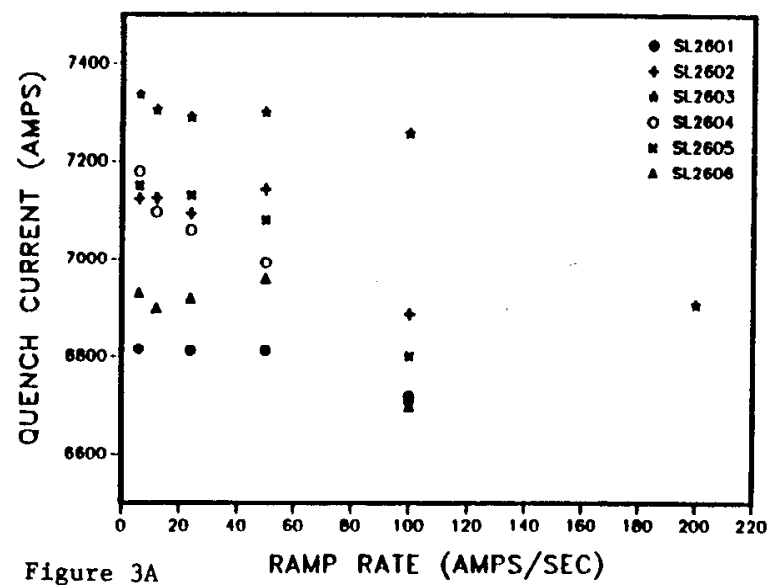

RAMP RATE DEPENDENCE OF QUENCH CURRENT

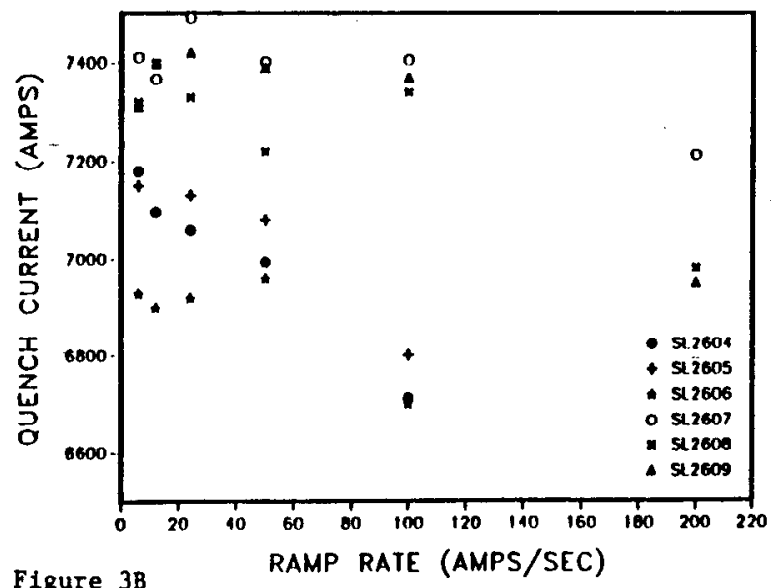

RAMP RATE DEPENDENCE OF OUENCH CURRENT

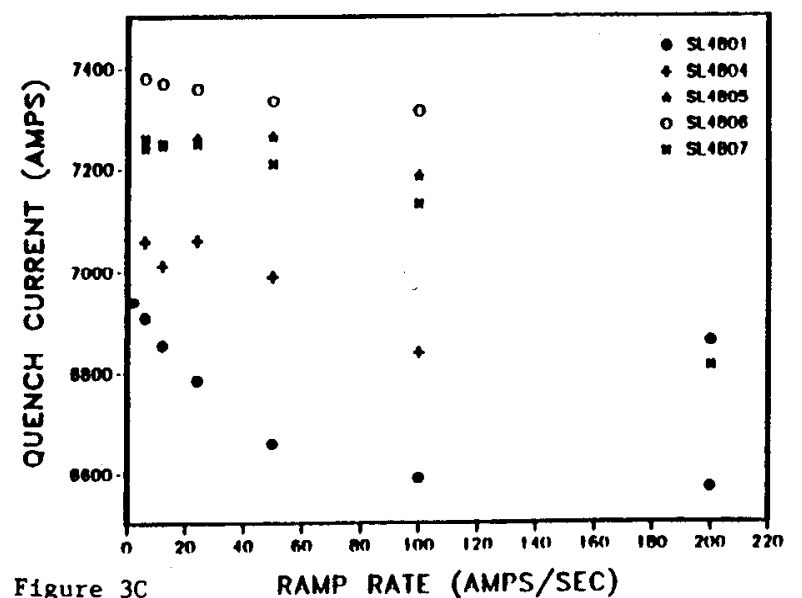

The energy loss due to eddy currents while ramping the magnet (Joules/cycle) is given in Table III. Typlcal data is shown in Figure 4. The $I^{2}$ curvature of the hysteresis cycle is due to the change in inductance of the magnet due to deflection caused by the Lorentz forces. The magnets are protected by quench heaters during the testing. The characterist10 7 ohm heater inputs required to quench the magnets powered to one klloamp were in the range of 460 Joules in 0.14 seconds to 280 Joules in 0.28 seconds.

Table III

EMERGY LOSS (JOULES)

CYCLE

50 - 5000 AMPS

MAGMEI RAMP RATE (AMPS/SEC)

\begin{tabular}{|c|c|c|c|c|c|c|}
\hline$\theta_{1} 2601$ & $\frac{6}{53.3}$ & $\frac{12}{58.9}$ & $\frac{24}{60.7}$ & $\frac{50}{62.7}$ & $\frac{100}{65.6}$ & $\frac{200}{68.4}$ \\
\hline$\theta_{1} 2602$ & 32.6 & 45.0 & 69.3 & 107.8 & 130.1 & 147.1 \\
\hline $0_{1} 2603$ & 33.1 & 46.4 & 74.0 & 113.2 & .131 .6 & 150.0 \\
\hline$a_{1} 2604$ & 34.2 & 41.4 & 56.9 & 89.8 & 108.8 & 128.8 \\
\hline $0_{1} 2605$ & 44.9 & 79.5 & 102.3 & 120.0 & 135.0 & 152.0 \\
\hline$\theta_{1} 2606$ & 27.3 & 53.2 & 89.8 & 116.1 & 134.1 & 148.4 \\
\hline $0_{1} 2607$ & 44.0 & 86.5 & & 148.1 & 147.5 & 160.8 \\
\hline $0_{1} 2608$ & 34.2 & 60.6 & 106.4 & 130.7 & 146.6 & 160.2 \\
\hline$o_{1} 2609$ & 42.4 & 59.6 & 99.8 & 127.2 & 149.0 & 157.1 \\
\hline$O_{2} 4801$ & 42.2 & 68.0 & 85.9 & 95.5 & 102.5 & 108.4 \\
\hline$O_{2} 4804$ & 54.6 & 85.5 & 121.9 & 146.5 & 166.1 & \\
\hline$O_{2} 4805$ & 64.0 & 136.4 & 172.9 & 193.5 & 211.8 & .234 .0 \\
\hline $\mathrm{O}_{2} 4806$ & & 137.2 & 165.8 & 189.9 & 210.0 & 240.0 \\
\hline$\theta_{2} 4307$ & 93.9 & 150.4 & 183.9 & 205.2 & 218.5 & 241.0 \\
\hline
\end{tabular}

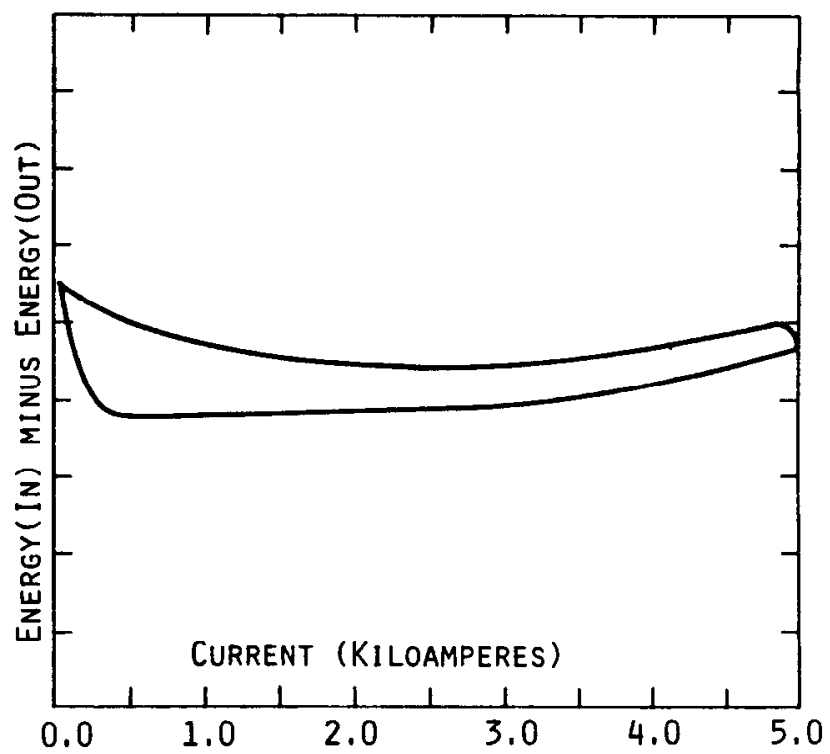

FIGURE 4 THE ENERGy (IN)-ENERGy (OUT) CURVE FOR SLO 2 4804. NOTE THE CURVATURE OF THE DATA INDICATING A CHANGE IN THE INDUCTANCE AT DEFLECTION OF THE COIL. 
Magnetic Characteristics

The integral strength and harmonics were first measured at room temperature by powering the quadrupoles with 5, 10, 15, and 20 amps, using a rotating $1.68 \mathrm{~m}$ long "Morgan" temperature relative strengths are given in Table IV using the strength of SLQ $\$ 4801=1$. The actual measured rat10 was $1.815+.066$ (Spec. was 1.821 ).

Table IV

\section{INTEGRAL RELATIVE STREHGTH} ROOM TEMPERATURE

\begin{tabular}{|c|c|c|c|c|c|}
\hline & 5 & 10 & 15 & 20 & av \\
\hline $4801 / 2601$ & $1.809+009$ & $1.213 \pm .001$ & $1.816 \pm .005$ & $1.812 £ .001$ & $1.812 \pm .003$ \\
\hline $4801 / 2602$ & $1.809 \pm .003$ & $1.814 \pm .003$ & $1.811 \pm .003$ & $1.812+.003$ & $1.811 \pm .002$ \\
\hline $4801 / 2603$ & $1.806 \pm .003$ & $1.808 \pm .003$ & $1.807 \pm .003$ & $1.807 \pm .003$ & $1.807 \pm .002$ \\
\hline $4801 / 2604^{\circ}$ & $1.809 \pm .004$ & $3.816 \pm .004$ & $1.810 \pm .004$ & $1.809 \pm .004$ & $1.811 \pm .004$ \\
\hline $4801 / 2605$ & $1.805 \pm .005$ & $1.311 \pm .003$ & $1.807 \pm .003$ & $1.805 \div .004$ & $1.807 \pm .003$ \\
\hline $4801 / 2606$ & $1.804 \pm .006$ & $1.811 \pm .005$ & $1.807 \pm .005$ & $1.803 \pm .004$ & $1.806 \pm .003$ \\
\hline $4801 / 2607^{\circ}$ & $1.824 \pm .008$ & $1.322 \pm .007$ & $1.819 \pm .006$ & $1.816 \div .005$ & $1.820 \pm .007$ \\
\hline $4801 / 2608^{\circ}$ & $1.810 \pm .005$ & $1.816 \pm .004$ & $1.808 \pm .004$ & $1.810 \pm .004$ & $1.811 \pm .004$ \\
\hline $4801 / 2609^{\circ}$ & $1.828 \pm .009$ & $1.818 \pm .006$ & $1.811 \pm .007$ & $1.810 \pm .004$ & $1.817 \pm .007$ \\
\hline $4801 / 4801$ & 1.0 & 1.0 & 1.0 & 1.0 & 1.0 \\
\hline $4801 / 4802$ & & $0.9972 \pm .01$ & & & \\
\hline $4801 / 4803$ & & $0.9965 \pm .002$ & & & \\
\hline $4801 / 4804$ & $0.9963 \pm .0015$ & $0.9975 \pm .0015$ & $0.9973 \pm .0015$ & $0.994 \pm .0015$ & $0,9963 \div .0015$ \\
\hline $4801 / 4805$ & $1.0037 \pm .0032$ & $1.0022 \pm .0034$ & $0.9998+.0032$ & $0.9974 \pm .0025$ & $1.0008 \pm .0028$ \\
\hline $4801 / 4806$ & $1.0003 \pm .0024$ & 1.0009 .0024 & $0.9992 \pm .0024$ & $0.9971 \pm .0024$ & $0.9994 \pm .0024$ \\
\hline 4801/4807 & $0.9997 \pm .0024$ & $1.0004 \div .0024$ & $0.9938 \pm .0024$ & $0.9972 \pm .0024$ & $0.9990 \pm .0024$ \\
\hline
\end{tabular}

SLQ 24801 - Integral Strength (20 mpS) per Mmp $=0.034 \pm .00007$ I GH CALCULATED tRansfer Function $=2.793$ GAUSS

- all readings to date

The $1.68 \mathrm{~m}$ long rotating coll was also inserted in the aperture when the magnet was at liquid helium temperatures. The integral gradient and the integral Fourler components of the fleld were measured at 2000 , 4000 and 4750 amperes DC. The ramped harmon1c content of the magnet ffeld was also measured. Figure 5 is a curve of duodecapole as a function of magnet current. The cold strengths of the various quadrupoles are given in Table $v$ in terms of $(\mathrm{Tesla} / \mathrm{cm})(\mathrm{cm})$.

The integral normalized harmonics at $2 \mathrm{kA}$ and $4 \mathrm{kA}$ are given in Table VI. These harmonics are given at $1 \mathrm{~cm}$ radius and are normalized by the gradient at that current, f.e., the sextupole term normalized at $I_{1}$, 1s given by:

$$
\frac{B_{2}\left(I_{1}, \frac{\text { Gauss }}{\mathrm{cm}^{*} \mathrm{~cm}}\right)}{B_{1}\left(I_{1}, \frac{\text { Gauss }}{\mathrm{cm}}\right)}=B\left(\text { Norm }_{2}\right)\left(I_{1}, 1 / \mathrm{cm}\right)
$$

The center of a quadrupole is defined by the condition $A_{0}$ and $B_{0}=0,1 . e .$, no dipole fleld.

PROBE $3 \quad 12$ POLE SL2603.

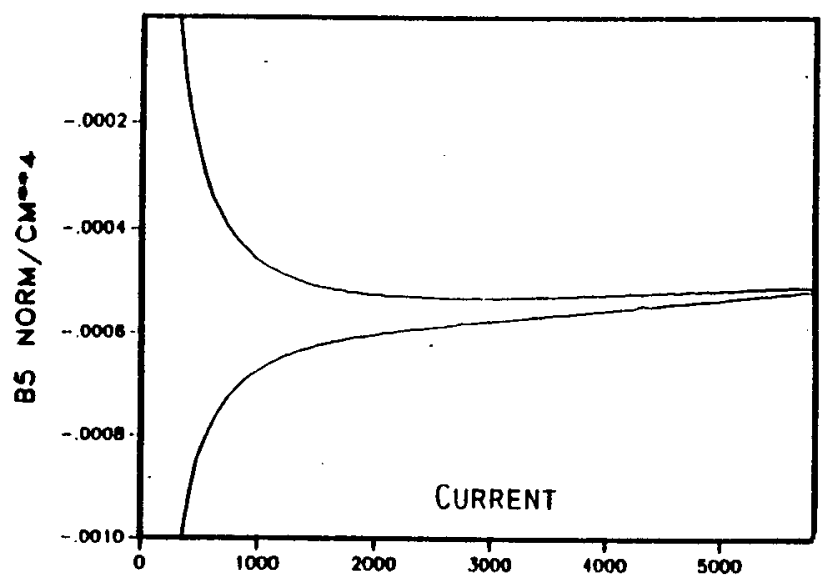

Figure 5 THE DUODECAPOLE (NORMALIZED) IS SHOWN AS A FUNCTION OF CURRENT FOR SLQ 2603 FOR A 6000 AMP TRIANGULAR CURRENT PULSE AS 6 AMPS/SEC

Table V

INTEGRAL STRENGTH ( $\left.\frac{\text { IESLA }}{\mathrm{CM}} \cdot \mathrm{CM}\right), 4.2 \mathrm{~K}$ 2000 AMPS $37.38 \pm .10$ $37.47 \pm .04$ $37.52 \pm .03$ $37.61 \pm .05$ $37.43 \pm .07$ $37.49 \pm .13$ $37.42 \pm .04$ $37.44 \pm .08$ $37.55 \pm .06$ $68.11 \pm .15$ $68.37 \pm . .10$ $68.05 \pm .15$ $68.11 \pm .27$ $68.03 \pm .14$ 4000 AMPS $75.06 \pm .13$ $75.07 \pm .10$ $75.08 \pm .11$ $74.93 \pm .15$ $75.06 \pm .19$ $74.96 \pm .09$ $75.06 \pm .12$ $75.21 \pm .10$ $136.21 \pm .44$ $136.71 \pm .33$ $136.21 \pm .45$ $136.30 \pm .20$

4806

4807

GRADIENT (TESLA/CM) $4.2 \mathrm{~K}$
2601

2602

2603

2604

2605

2606

2607

2608

2609

4801

4804

4805

4806

4807
$0.5614 \pm .004$

$0.5617 \pm .0013$

$0.5624 \pm .0014$

$0.5643 \pm .0014$

$0.5606 \pm .0011$

$0.5611 \pm .0015$

$0.5606 \pm .0003$

$0.5606 \pm .0005$

$0.5618 \pm .0004$

$0.5613 \pm .0018$

$0.5635 \pm .0016$

$0.5604 \pm .0011$

$0.5609 \pm .0013$

$0.56-9 \pm .0011$
$1.120 \pm .002$

$1.121 \pm .004$

$1.126 \pm .003$

$1.122 \pm .002$

$1.123 \pm .002$

$1.122 \pm .001$

$1.123 \pm .001$

$1.125 \pm .001$

$1.123 \pm .003$

$1.122 \pm .002$

$1.123 \pm .002$

$1.122 \pm .002$
4750 AMPS

$88.99 \pm .11$ $89.12 \pm .12$ $88.99 \pm .18$ $89.17 \pm .23$ $89.11 \pm .12$ $89.17 \pm .23$ $89.38 \pm .12$ $161.40 \pm .50$ $162.00 \pm .38$ $161.78 \pm .44$ $161.85 \pm .29$ $161.50 \pm .29$
$1.331+.004$

$1.335 \pm .003$

$1.332 \pm .003$

$1.333 \pm .002$

$1.333 \pm .001$

$1.333 \pm .001$

$1.336 \pm .001$

$1.333 \pm .003$

$1.333 \pm .005$

$1.334 \pm .005$

$1.333 \pm .004$ 
Norellized Integral Harwonics

$\times 10^{\circ}$

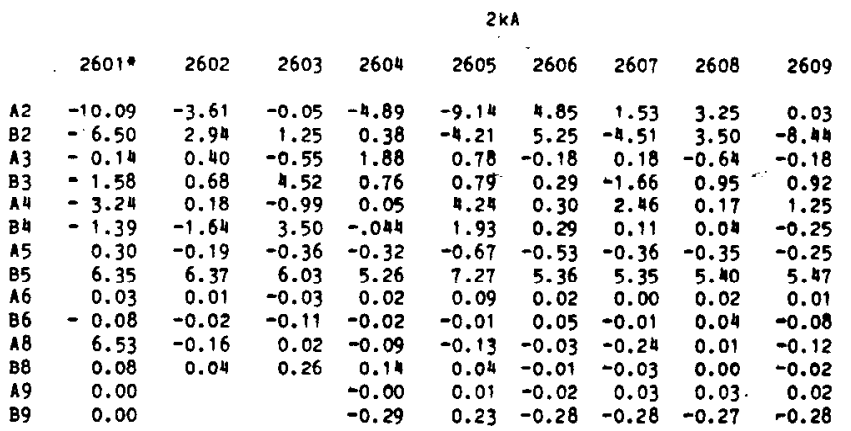

- Data taken at 1 k1loamp

4 ike

12
82
13
83
14
84
15
95
16
86
18
88
19
89

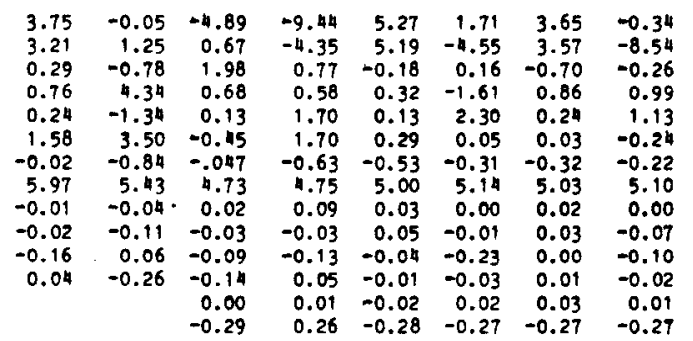

Mormallzed Integral Harmonics

$\times 10^{*}$

$2 k h$

\begin{tabular}{|c|c|c|c|c|c|}
\hline & 4801 & 4804 & 4805 & 4806 & 4807 \\
\hline 12 & -0.49 & 3.25 & 2.17 & -4.63 & 0.29 \\
\hline 82 & 4.49 & -3.25 & 2.56 & -3.38 & 1.64 \\
\hline A3 & 1.42 & -0.39 & -0.43 & -0.32 & -0.66 \\
\hline B3 & 2.40 & -1.05 & 0.05 & 0.48 & -0.69 \\
\hline 14 & -0.67 & 0.43 & -0.09 & 2.14 & 2.20 \\
\hline B4 & -2.96 & -0.58 & -0.16 & -0.38 & -0.24 \\
\hline 15 & -0.26 & 0.73 & -0.42 & -0.11 & -0.38 \\
\hline 85 & 5.61 & 2.71 & 5.10 & 5.27 & 5.42 \\
\hline 16 & 0.37 & 0.04 & 0.01 & -0.04 & -0.00 \\
\hline B6 & 0.38 & 0.01 & -0.02 & 0.02 & -0.00 \\
\hline 18 & 0.04 & -0.01 & -0.19 & $=0.19$ & -0.29 \\
\hline BB & 0.48 & -0.08 & 0.14 & 0.20 & -0.04 \\
\hline 19 & -0.18 & -0.01 & -0.02 & -0.02 & 0.03 \\
\hline B9 & -0.21 & -0.29 & -0.28 & -0.28 & -0.28 \\
\hline & - & \multicolumn{4}{|c|}{$4 k A$} \\
\hline 12 & & 3.67 & 2.42 & -4.66 & 0.32 \\
\hline 82 & & -4.02 & 2.67 & -3.39 & 1.70 \\
\hline 13 & & -0.39 & -0.52 & -0.31 & -0.66 \\
\hline B3 & & -1.24 & 0.06 & 0.48 & -0.63 \\
\hline A4 & & 0.36 & -0.13 & 2.00 & 2.10 \\
\hline 84 & & -0.75 & -0.18 & -0.35 & -0.25 \\
\hline 15 & & -0.77 & -0.42 & -0.10 & -0.38 \\
\hline B5 & & 3.30 & 4.93 & 5.10 & 5.16 \\
\hline 16 & & -0.65 & 0.01 & -0.05 & -0.00 \\
\hline B6 & & -0.95 & -0.02 & 0.01 & -0.00 \\
\hline A8 & & -0.01 & -0.19 & -0.19 & -0.29 \\
\hline B8 & & -0.08 & 0.15 & 0.20 & 0.23 \\
\hline 19 & & $=0.01$ & -0.02 & -0.02 & -0.03 \\
\hline B9 & & -0.33 & -0.28 & -0.28 & -0.28 \\
\hline
\end{tabular}

The next set of data of Interest are the straight section field strengths and harmonic content. The magnet design was originally done to minimize the varlous non quadrupole components of the field. In Table VII, the body fleld normalized Fourler components are given for $2 \mathrm{kA}$ and for $4 \mathrm{kA}$.
$2 k A$

$\begin{array}{lrrrrrrrrr} & 2601 & 2602 & 2603 & 2604 & 2605 & 2606 & 2607 & 2608 & 2609 \\ A 2 & -5.14 & -0.69 & -0.39 & 0.93 & -4.71 & -0.98 & -1.83 & 1.63 & -1.14 \\ B 2 & -1.72 & 0.09 & 0.17 & -2.34 & -0.63 & 2.10 & -2.26 & -1.82 & -2.02 \\ \text { A3 } & 0.15 & 0.32 & 0.50 & -0.98 & -0.09 & -0.03 & 0.19 & 0.33 & 0.16 \\ B 3 & 0.54 & -1.10 & 1.48 & -0.72 & 0.68 & 0.74 & 1.74 & -0.46 & -0.78 \\ A 4 & 0.65 & 0.49 & 0.64 & -0.47 & -0.17 & -0.13 & 0.15 & -0.01 & -0.07 \\ B 4 & 1.07 & 0.59 & 2.63 & 1.29 & 1.02 & 0.71 & -0.95 & -2.30 & 0.94 \\ A 5 & -0.60 & 0.09 & 0.00 & 0.05 & -0.63 & -0.33 & -0.24 & -0.22 & -0.11 \\ B 5 & 5.87 & 6.51 & 6.01 & 4.32 & 0.82 & 5.22 & 5.44 & 5.37 & 5.27 \\ A 6 & -0.16 & 0.02 & -0.02 & 0.04 & -0.09 & 0.00 & 0.03 & 0.00 & 0.04 \\ B 6 & 0.70 & 0.21 & 0.10 & -0.02 & 0.03 & -0.01 & 0.01 & 0.03 & 0.05 \\ A B & 5.11 & 0.10 & -0.03 & -0.03 & 0.07 & 0.10 & -0.38 & 0.13 & -0.05 \\ B 8 & 0.30 & 0.02 & 0.44 & 0.13 & 0.75 & 0.11 & 0.18 & 0.10 & -0.07 \\ A 9 & -0.02 & 0.00 & -0.01 & -0.12 & 0.25 & 0.00 & 0.12 & 0.27 & -0.76 \\ B 9 & -0.33 & -0.34 & -0.34 & -0.23 & -0.24 & -0.31 & -1.12 & 0.27 & 0.11 \\ A 13 & 0.00 & 0.00 & 0.00 & 0.01 & -0.02 & 0.00 & & & 1 \\ B 13 & 0.00 & 0.01 & 0.00 & 0.02 & 0.15 & 0.00 & & & \end{array}$

AkA

12
82
13
83
14
$B 4$
15
85
16
86
18
88
19
89
113
$B 13$

$\begin{array}{llllllll}-0.46 & -0.38 & 0.80 & -4.95 & -0.60 & -1.73 & 1.70 & -1.36\end{array}$ $\begin{array}{llllllll}0.17 & 0.12 & -2.07 & -0.98 & 2.39 & -2.59 & -2.06 & -1.95\end{array}$ $\begin{array}{llllllll}0.43 & 0.55 & -1.07 & -0.07 & -0.03 & 0.24 & 0.41 & 0.24\end{array}$ $\begin{array}{llllllll}-1.22 & 1.42 & -0.69 & 0.71 & 0.69 & 1.72 & -0.50 & -0.78\end{array}$ $\begin{array}{llllllll}0.49 & -0.64 & -0.18 & -0.07 & -0.20 & 0.15 & 0.04 & -0.05\end{array}$ $\begin{array}{llllllll}0.63 & 2.43 & 1.18 & 1.04 & 0.74 & -0.86 & -2.09 & 0.90\end{array}$ $\begin{array}{llllllll}0.08 & 0.01 & 0.00 & -0.15 & -0.31 & -0.20 & -0.19 & -0.08\end{array}$ $\begin{array}{llllllll}6.08 & 5.55 & 4.86 & 0.08 & 4.81 & 5.09 & 5.05 & 4.85 \\ 0.02 & 0.07 & 0.05 & -0.09 & -0.01 & 0.03 & 0.00 & 0.04\end{array}$ $\begin{array}{llllllll}0.02 & 0.07 & 0.05 & -0.09 & -0.01 & 0.03 & 0.00 & 0.04 \\ 0.03 & 0.03 & -0.03 & -0.09 & -0.02 & 0.03 & 0.03 & 0.06\end{array}$ $\begin{array}{llllllll}0.03 & 0.03 & -0.03 & -0.09 & -0.02 & 0.03 & 0.03 & 0.06 \\ -0.11 & 0.08 & -0.14 & 0.16 & 0.10 & -0.35 & -0.04 & -0.02\end{array}$

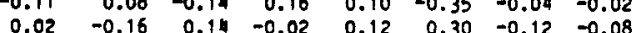
$\begin{array}{rrrrrrrr}0.02 & -0.16 & 0.11 & -0.02 & 0.12 & 0.30 & -0.12 & -0.08 \\ 0.00 & 0.00 & 0.26 & -0.43 & 0.02 & -0.14 & 0.19 & -0.72\end{array}$ $\begin{array}{rrrrrrrr}0.00 & 0.00 & 0.26 & -0.43 & 0.02 & -0.14 & 0.19 & -0.72 \\ -0.34 & -0.34 & -0.58 & -0.58 & -0.32 & -1.27 & 0.23 & -0.04\end{array}$ $\begin{array}{ll}0.01 & -0.00\end{array}$

$113=0.0004 \pm .002$

$013=-0.002 \pm .009$

Mormallzed Harmonloe stratght Section

$\times 10^{\circ}$

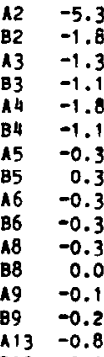

1. -0.88

$813-0.11$

$A 2$
82
13
83
14
84
15
85
16
86
18
88
19
89
113
$B 13$

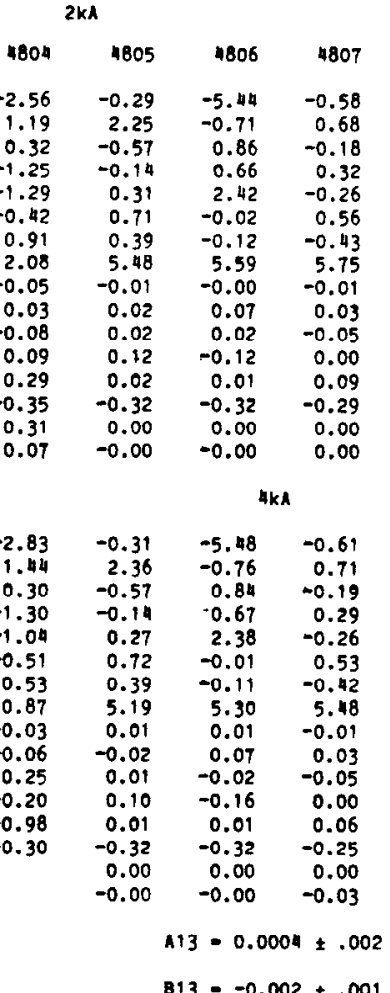


The Integral and straight section harmonics at the operational fleld current of 4759 Amperes is given In Appendix 1, Table $E$ and $F$.

There appeared to be a change in the normallzed duodecapole term of about a half a unit $\left(10^{-}\right)$in a time perlod of approximately 200 seconds at a magnet curent of 4000 Amps.

APPENDIX 1

Table A

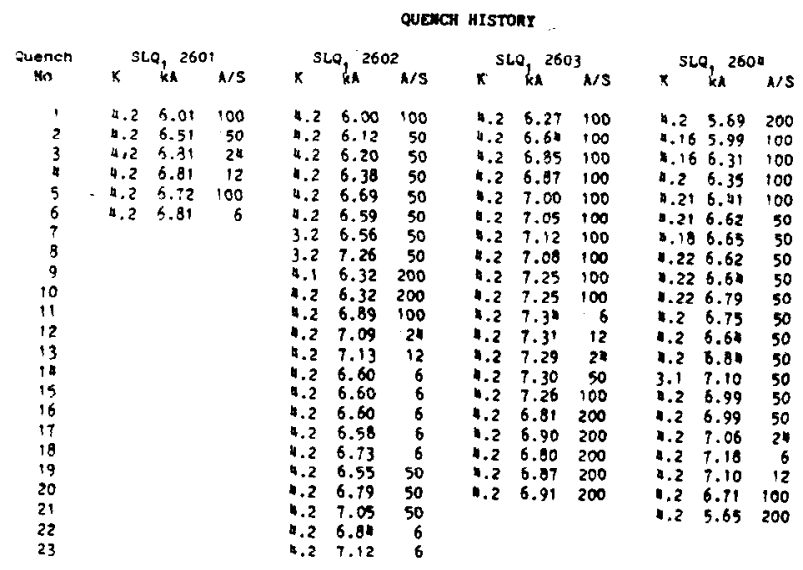

Table B
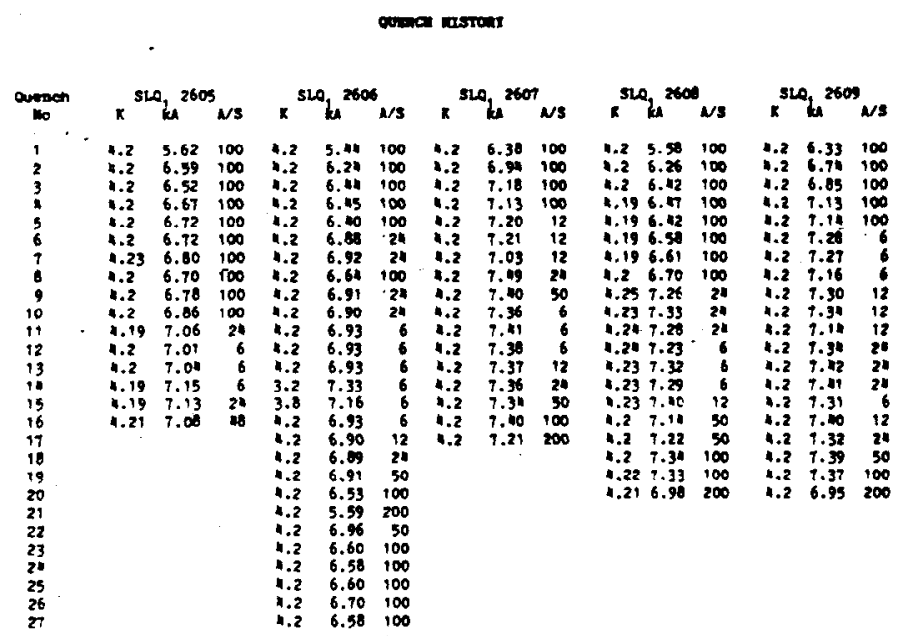

QVEMCH HISTORY

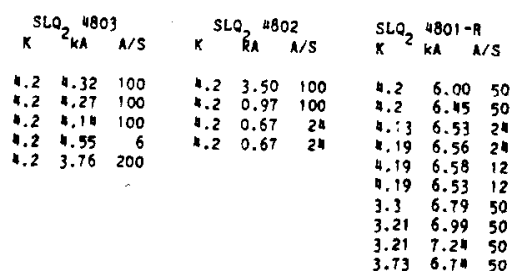

Table D
Table C
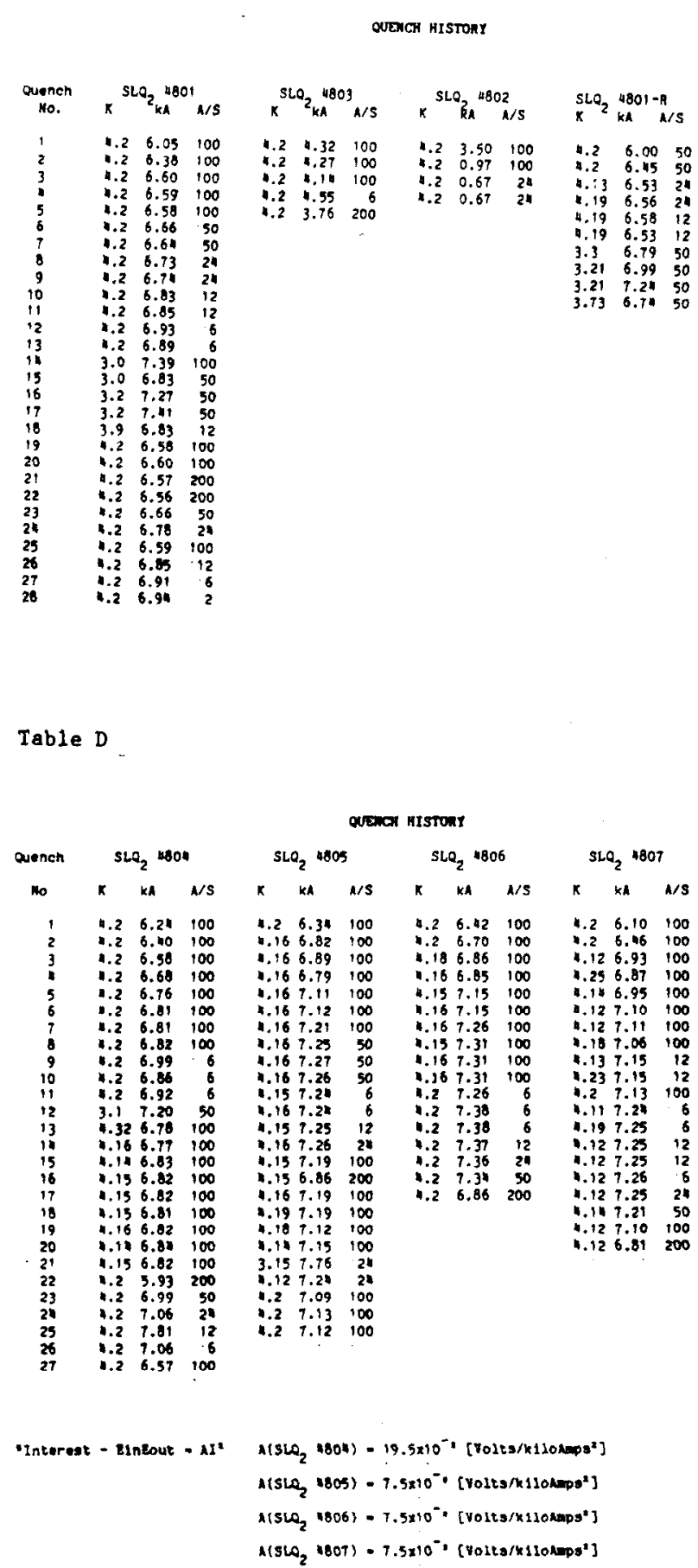


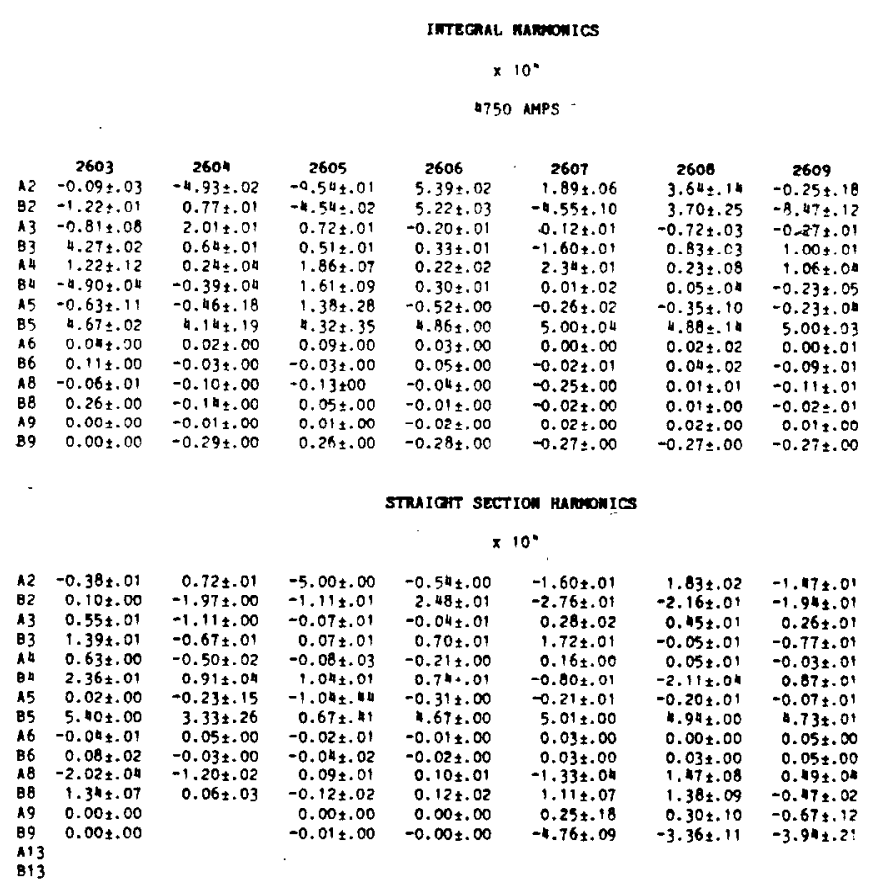

The authors would like to express their appreclation to the skilled techniclans who enabled this project to be successfully accompllshed: L. Davis, C. Diebold, A. Gllly, S. Gould, C. Hess, C. Iorlatt1, W. Mumper, R. Nehring, D. Nurczyk, R. Remsbottom, A. Rusy, G. Smith, R. Volgts, $H$. Warren, and $D$. Wendt.

\section{References}

[1] R. Ericksion, T. Fleguth, and J.J. Murray, "Superconducting Quadrupoles for the SLC Final Focus," SLAC-PUB-4199, January, 1987.

[2] R.A. Lundy, B.C. Brown, J.A. Carson, H.E. Fisk, R.H. Hanft, P.M. Mantsch, A.D. MeInturff, R.H. Remsbottom, and R. Ertckson, "High Gradient Superconducting Quadrupoles," IEEE TRANS. on Nuclear Sclence, Vol. NS-32, No. 5, pp. 3707 $3709,1985$.

[3] G.H. Morgan, "Stationary Co1l For Measuring the Harmonics in Pulsed Transport Magnets," Proc. 4th International Conf. on Magnet Technology, Brookhaven, Sept. 19-22, 1972, USAEC CONF-720908, NTIS, Springfield, Va., (1973) pp. $787-790$.

Table F

INTEGRAL HARMONICS

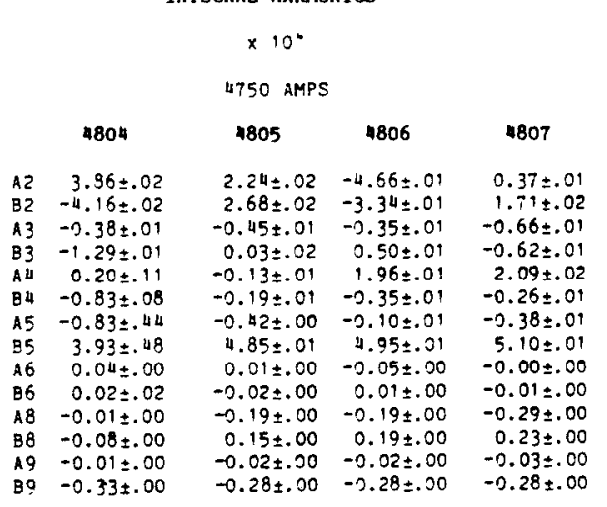

STRAIOHT SECTION HARMONICS

$$
\times 10^{\circ}
$$

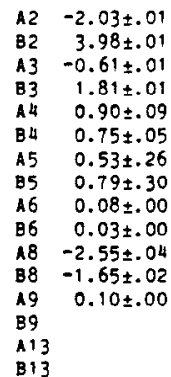

$\begin{array}{rrr}-0.33 \pm .01 & -5.50 \pm .01 & -0.64 \pm .01 \\ 2.37 \pm .00 & -0.77 \pm .01 & 0.72 \pm .01 \\ -0.59 \pm .00 & 0.82 \pm .09 & -0.18 \pm .01 \\ -0.15 \pm 01 & 0.68 \pm .10 & 0.29 \pm .00 \\ 0.27 \pm .01 & 2.38 \pm .01 & -0.26 \pm .00 \\ 0.75 \pm .01 & -0.02 \pm .01 & 0.54 \pm .01 \\ 0.38 \pm .00 & -0.11 \pm .00 & -0.42 \pm .01 \\ 5.12 \pm .00 & 5.23 \pm .00 & 5.42 \pm .00 \\ 0.01 \pm .00 & 0.01 \pm .00 & -0.01 \pm .00 \\ -0.02 \pm .00 & 0.07 \pm .00 & 0.03 \pm .00 \\ 0.09 \pm .01 & -1.40 \pm .02 & -0.35 \pm .01 \\ 1.00 \pm .02 & 1.63 \pm .03 & 0.08 \pm .01 \\ 0.01 \pm .00 & 0.01 \pm .00 & \\ -0.32 \pm .00 & -0.32 \pm .00 & -0.28 \pm .02 \\ 0.00 \pm .00 & 0.006 \pm .00 & 0.00 \pm .00 \\ 0.00 \pm .00 & -0.002 \pm .00 & 0.00 \pm .00\end{array}$

